\title{
Clinical Trials Corner Issue 7(3)
}

Piyush K. Agarwal ${ }^{\mathrm{a}, 1}$ and Cora N. Sternberg ${ }^{\mathrm{b}, 1}$

${ }^{a}$ The University of Chicago, Chicago, IL, USA

${ }^{\mathrm{b}}$ Englander Institute for Precision Medicine, Weill Cornell Medicine, Meyer Cancer Center, New York, NY, USA

Received 14 July 2021

Accepted 18 July 2021

Pre-press 16 August 2021

Published 31 August 2021

Dear Readers,

In this issue, we highlight several trials evaluating bladder preservation. While exciting, these trials do raise the question of comparability across studies given the use of different regimens of radiotherapy and different endpoints. In the future, please reach out to us directly in order to highlight any specific clinical trials at pkagarwal@uchicago.edu or cns9006@med.cornell.edu and/or at BLC@iospress.com.

Sincerely,

Piyush K. Agarwal, MD

Associate Editor, Bladder Cancer

Director, Bladder Cancer Program

The University of Chicago

Chicago, Illinois
Cora N. Sternberg, MD, FACP

Associate Editor, Bladder Cancer

Clinical Director, Englander Institute for Precision Medicine

Weill Cornell Medicine

New York, New York

Study Title: Pembrolizumab (MK3475), Gemcitabine, and Concurrent Hypofractionated Radiation Therapy for Muscle-Invasive Urothelial Cancer of the Bladder

\section{Clinicaltrials.gov identifier: NCT02621151}

Sponsor: Merck

\section{Enrollment: 54}

Rationale: Chemoradiation is an established treatment for MIBC and this trial is evaluating whether the addition of immunotherapy, specifically, pembrolizumab can improve upon this response. Furthermore, the trial uses an unique endpoint of intact bladder-free survival which minimizes the impact of urinary symptoms and/ or recurrence of NMIBC.

Study Design: This is a single arm Phase I/II trial evaluating the safety of the combination of pembrolizumab, gemcitabine, and radiation therapy with an initial safety lead-in cohort of 6 patients. This will be followed by a phase II cohort if toxicity is only seen in 1 or zero patients consisting of 48 patients for efficacy evaluation. The results were presented at ASCO 2021. The chemoradiation consisted of whole bladder radiation therapy (52 Gy in 20 fractions over 4 weeks) with twice weekly gemcitabine at $27 \mathrm{mg} / \mathrm{m}^{2}$ for 4 weeks given concurrent with chemoradiation. Pembrolizumab was given as a single lead-in dose $(200 \mathrm{mg})$. Treatment was followed by

${ }^{1}$ Contributed equally. 
maximal TURBT 2- 3 weeks afterwards and pembrolizumab $200 \mathrm{mg}$ intravenously was administered every 3 weeks for a total of 3 doses starting day 1 of radiation therapy.

Endpoints: The primary endpoint was two-year bladder intact event-free survival (BI-EFS) which is a novel endpoint only measured from the time of treatment initiation to the following events: recurrent/residual MIBC post-chemoradiotherapy, nodal or distant metastatic disease, radical cystectomy, or death due to any cause. Secondary endpoints included safety, complete response rate, overall survival and metastasis-free survival. Complete response rate was defined as the absence of any viable residual cancer.

Results: Updated results were presented at ASCO 2021. Six patients were enrolled into a safety cohort followed by 48 patients enrolled into an efficacy cohort. A total of 42 patients (85\%) completed all protocol therapy and were included in the analysis. The majority of patients had T2N0 disease $(70 \%)$ and $75 \%$ refused cystectomy. At 12 weeks post-radiotherapy, complete response in the entire cohort was 59\%. At a median follow-up of 14.6 months, bladder-intact free survival was $88 \%$ at 12 months in the efficacy cohort. Nine patients (19\%) were treated with steroids for immune-related adverse events. One patient died of a grade 4 colonic perforation.

Comments: The initial results are exciting given the complete response rate and high bladder-intact free survival rate, albeit with short follow-up. Of course with further follow-up, these numbers can worsen over time but the regimen appears to be safe and outcomes are initially quite reasonable. Another challenge will lie in knowing the ideal radio-sensitizing chemotherapy agent to use along with the best radiation treatment protocol. To that end, the ongoing randomized clinical trial evaluating the use of pembrolizumab with investigator choice of chemotherapy and radiation therapy (NCT 04241185) is actively accruing and will answer more questions.

\section{Study Title: Gemcitabine, Cisplatin, Plus Nivolumab in Patients With Muscle-invasive Bladder Cancer With Selective Bladder Sparing (GU-16-257)}

\section{Clinicaltrials.gov identifier: NCT03558087}

Sponsor: Icahn School of Medicine at Mount Sinai/Hoosier Cancer Research Network

\section{Enrollment: 76}

Rationale: This trial explores the impact of primary chemo-immunotherapy as a sole-treatment for MIBC without the use of radiotherapy. Therefore, this trial is evaluating the use of systemic therapy for localized disease without the use of definitive local therapy.

Study Design: This is a single arm, phase II study in which cisplatin-eligible patients with cT2-T4aN0M0 urothelial cancer are treated with TURBT followed by four cycles of gemcitabine/cisplatin and nivolumab and patients reassessed with clinical imaging, cytology, cystoscopy, and biopsies and if patients achieved a clinical complete response rate, then offered a four-month adjuvant period of nivolumab instead of radical cystectomy. Clinical complete response rate is defined as normal imaging, normal cytology, and no tumor or low grade Ta urothelial cancer on biopsy.

Endpoints: The primary endpoints are clinical complete response rate (cT0 or cTa) and the ability of clinical complete response to predict pathologic complete response $(<\mathrm{pT} 1)$ in patients undergoing cystectomy and 2 year metastasis-free survival (MFS) in patients pursuing surveillance. Secondary endpoints include the impact of baseline genomic alterations from TURBT (ERCC2, FANCC, RB1, ATM, and tumor mutation burden) on performance of clinical complete response for predicting MFS.

Results: Overall, 76 patients were enrolled and 64 (84\%) completed imaging. Thirty-one (48\%) of the 64 patients achieved a complete response and 30 of the 31 patients chose adjuvant immunotherapy instead of 
radical cystectomy. However, 8 of the 31 patients developed a local recurrence and six of them underwent cystectomy and only one patient had $>\mathrm{T} 2 \mathrm{~N} 0$ disease. Of note, among the patients without complete response who underwent immediate cystectomy $(\mathrm{n}=28), 32 \%$ had ypTanyN+ disease. Finally, among genomic alterations well described to correlate with response to chemoimmunotherapy, only TMB $\geq 10$ mutations/Mb or mutant ERCC2 were associated with a clinical complete response.

Comments: TURBT alone for MIBC has been shown in some series to show excellent response rates and in this study the rates of clinical complete response are lower than historical TURBT series and certainly lower than in studies with definitive local therapy using chemoradiation or radical cystectomy. Furthermore, genomic alterations known to correlate with response to chemoimmunotherapy may not function as biomarkers as they may not correlate with clinical response in all patients.

\section{Study Title: Phase III Randomized Trial of Concurrent Chemoradiotherapy With or Without Atezolizumab in Localized Muscle Invasive Bladder Cancer}

\section{Clinicaltrials.gov identifier: NCT03775265}

Sponsor: SWOG/NRG (National Cancer Institute), Genentech

\section{Enrollment: 475}

Rationale: Chemoradiation has an established therapeutic role in localized muscle-invasive bladder cancer. This cooperative group trial tests the hypothesis that the addition of immunotherapy, specifically atezolizumab, improves bladder intact event-free survival.

Study Design: This is a randomized trial for patients with localized muscle-invasive bladder cancer T24N0M0 who have refused or are unfit for radical cystectomy or preferentially choose bladder sparing treatment whereby patients are randomized to radiation therapy (three-dimensional conformal or intensity-modulated) with chemotherapy as per the treating physician with or without atezolizumab. The chemotherapy can consist of either gemcitabine, cisplatin, or fluorouracil and mitomycin. Patients treated in the atezolizumab arm are treated every 3 weeks for up to 6 months.

Endpoints: The primary outcome is bladder intact event-free survival (BI-EFS) rate from the date of randomization to the first documentation of a BI-EFS event, assessed up to 5 years .

This composite endpoint includes the absence of muscle invasive bladder recurrence, regional pelvic soft tissue or nodal recurrence, distant metastases, bladder cancer or toxicity related death or cystectomy. Secondary endpoints include: overall survival at 5 years, disease-specific survival, NMIBC recurrence rate, cystectomy rate, and several immunologic and biologic endpoints.

Results: At GU ASCO 2021, safety data were reported for the first 73 patients enrolled on trial. The atezolizumab arm contained 37 patients while the trimodal therapy arm contained 36 patients. Overall, the immunotherapy arm had 23 grade 3 or higher toxicity rates compared to 11 in the trimodal therapy alone arm. Moreover, none of the toxicities were deemed to be immune-related. The most common toxicity was hematological (anemia, lymphopenia and decreased WBC).

Comments: This is an ongoing trial with a target enrollment of 475 patients. As of July 1,2021 172 patients have been registered with a target enrollment of 10 patients per month. The DSMC considered it safe to continue the trial, though it is surprising that there were more grade 3 or high toxicity events in the immunotherapy arm that were deemed not to be immune-related. This suggests that combination therapy may result in a higher number of toxicities that are a result more of combination treatment as opposed to being related to immunotherapy alone. 


\section{CONFLICT OF INTEREST}

\section{Cora N. Sternberg}

Consultant: Pfizer, Merck Ga, AstraZeneca, Astellas Pharma, Sanofi-Genzyme, Roche/Genentech, BMS, Medscape, Immunomedics, Clovis Oncology, UroToday, MSD, Foundation Medicine

\section{Piyush K. Agarwal}

Advisory Board (unpaid): AstraZeneca; Advisory Board (paid): AURA, Verity

\section{References:}

1. Powles T et al, N Engl J Med, 2021 Mar 25;384(12):1125-1135. doi: 10.1056/NEJMoa2035807.Epub 2021 Feb 12.

2. Bajorin D et al, ASCO GU, Abstract Number 391

3. Bellmunt J et al, Lancet Oncol. 2021 Apr;22(4):525-537. doi: 10.1016/S1470-2045(21)00004-8. Epub 2021 Mar 12.

4. Apolo A, ASCO GU 2019, abstract 